\title{
ANEMIA APLÁSTICA SEVERA: ANÁlISE dOS PACIENTES PEDIÁTRICOS ATENDIDOS PELO SERVIÇO DE TRANSPLANTE DE MEDULA ÓSSEA DO HOSPITAL DE CLÍNICAS DE CURITIBA NO PERÍODO DE 1979-1993
}

Teresa Cristina Cardoso fonseca*, Ricardo Paquini

Trabalho realizado no Departamento de Pediatria do Hospital de Clínicas da Universidade Federal do Paraná, Curitiba, PR.

RESUMO - A anemia aplástica severa é uma doença rara que acomete pessoas de todas as regiöes do mundo. Devido a sua raridade, existem dificuldades em se caracterizar 0 perfil dos pacientes acometidos pela doença, assim como identificar possíveis fatores implicados na sua etiologia.

ObjETVO. Descrever o perfil dos pacientes pediátricos atendidos com anemia aplástica severa, analisando variáveis como distribuição de idade, sexo, local de habitação, etiologia e nível socioeconômico.

Ḿ́todos. Foi realizada uma análise descritiva das características encontradas em todos os pacientes com idade inferior a 18 anos, encaminhados no período de 1979-1993 com diagnóstico da doença, conforme classificação proposta por Camitta et al. As informaçöes foram obtidas através do prontuário médico e de um questionário aplicado pela pesquisadora em 79 das 134 famílias.

Resultados. A média das idades foi de 10,89 anos. Houve leve predominância do sexo feminino. A maioria dos pacientes residia na zona urbana da regiäo sul do país. $34 \%$ das famílias não identificaram um fator causal. Os pesticidas agrícolas e os derivados de benzeno foram os fatores causais mais relatados no questionário. Das 79 familias que responderam ao questionário, 83,5\% tinham renda familiar inferior a I salário mínimo "per capita" e $70,9 \%$ das mães tinham nível de escolaridade inferior 0 primeiro grau completo.

Conclusão. 0 perfil da população pediátrica com anemia aplástica severa atendida no Serviço de Transplante de Medula Ossea de Curitiba é formado predominantemente por crianças de II anos, do sexo feminino, provenientes da zona urbana da regiäo sul do país, com baixo nível socioeconômico, que relataram os pesticidas agrícolas e derivados de benzeno como possíveis fatores etiológicos.

Unitermos: Anemia aplástica severa. Etiologia. Nível socioeconômico. Brasil.

\section{INTRODUÇÃO}

A anemia aplástica severa é uma doença hematológica rara, de elevada letalidade, freqüentemente de causa desconhecida e encontrada nas diversas regiões no mundo. Estudos populacionais demonstram que a doençaé mais comum no oriente do que no ocidente. $\mathrm{Na}$ Tailândia, desde 1989, está sendo realizado um estudo caso-controle sobre fatores de risco para anemia aplástica e sua incidência, e este grupo demonstrou um coeficiente de incidência para cidade de Bangkok de 3,9 casos novos/1000000 habitantes por ano, $0^{1,2}$. Já no ocidente (Europa e lsrael) desde 1980 foi realizado o maior e um dos mais controlados estudos sobre anemia aplástica e agranulocitose - "International Aplastic Anemia and Agranulocytosis Study". Neste estudo, houve um coeficiente de incidência de dois casos

*Correspondencia:

Rua Rio Colônia, 307/100-Góes Calmon CEP 45605-900 - Itabuna - Bahio novos/ 1000000 habitantes por ano $0^{3,4}$. Registros hospitalares sugerem uma grande incidência da doença na América Latina, no entanto, no Brasil, um estudo no estado do Paraná teve um coeficiente de incidência de 2,4 casos/ 1000000 habitantes por ano ${ }^{5}$.

A distribuição da doença em relação ao sexo, idade e localização geográfica varia nos diversos estudos epidemiológicos 6 . Os possíveis fatores etiológicos presentes no ambiente poderão desencadear a doença conforme a intensidade da exposição da população a esses fatores, assim como o seu perfil genético. Alguns inquéritos descreveram uma maior incidência da doença em grupos populacionais de baixo nível socioeconômico ${ }^{7,8}$.O nível socioeconômico não seria um fator etiológico, mas provavelmente expressaria uma exposição a determinados agentes ambientais que não tenham sido identificados, como por exemplo, um agente infeccioso de contaminação oral-fecal.

Em termos etiológicos, vários são os agentes causais implicados no aparecimento da anemia aplástica. Estão incluídos nesse grupo agentes químicos, físicos e infecciosos. Dentre os agentes químicos encontrados na comunidade eque regularmente produzem a depressão medular estão os derivados de benzeno. Os inseticidas, anticonvulsivantes, antiinflamatórios, antimaláricos, cloranfenicol e metais pesados são drogas que possivelmente estão relacionados com a aplasia medular?

Dos agentes infecciosos, estudos epidemiológicos relacionaram história pregressa dehepatite e de mononucleose infecciosa com o aparecimento da anemia aplástica ${ }^{10,11,12}$. Existem relatos de casos do desencadeamento da doença, após infecçãopelo citomegalovírus evírus da dengue ${ }^{13}$.

A radiação ionizante, apesar de ser um agente relacionado com a aplasia medular, não é um agente que freqüentemente é encontrado na comunidade ${ }^{14}$.

O objetivo deste estudo é descrever a população pediátrica atendida com anemia aplástica severa no Serviço de Transplante de Medula Óssea do Hospital de Clínicas da Universidade Federal do Paraná, atendida no período de 1979 até o ano de 1993, analisando 
variáveis como a distribuição da doença em relação ao sexo, idade, procedência, local de habitação, etiologia e nível socioeconômico.

\section{Métodos}

Foram analisados os cadastros de todas as crianças com o diagnóstico de anemia aplástica severa conforme a classificação de Camitta etal. ${ }^{15}$ do ano de 1979-1993. Elaborou-se um questionário que foi aplicado no ano de 1994 e 1995 pela pesquisadora aos familiares dos pacientes contactados. Oquestionário foi dividido em três partes: dados pessoais, etiologia e avaliação socioeconômica. Em termos etiológicos, foram investigados a etiologia viral (hepatite, mononucleose, dengue e citomegalovírus), medicamentos (cloranfenicol, sais de ouro, anticonvulsivantes, antimaláricos e antiinflamatórios), pesticidas agrícolas, derivados de benzeno (solventes, cola de sapateiro, tintas e produtos petroquímicos)eradiaçãoionizante. Foiconsideradaagenteresponsável naetiologia da doença, a medicação quefoiutilizada atéseis meses anteriores ao aparecimento da doença, e, em termos infecciosos, os agravos que ocorreram até seis meses anteriores ao seu diagnóstico.

Com relação aos pesticidas agrícolas, aos derivados de benzeno e a radiação ionizante, só foram considerados os eventos nos quais as crianças tiveram uma toxicidade aguda ou as que conviveram em uma área com este material por pelo menos 15 dias nos últimos seis meses do diagnóstico.

A avaliação socioeconômica foi baseada na renda familiar "per capita" e na escolaridade materna. Utilizou-se a renda familiar de um salário mínimo "per capita" como nível divisório, pois segundo o DIEESE (Departamento Intersindical de Estatística e Estudos Socioeconômicos), este é o nível mínimo de renda compativel com as necessidades básicas de uma família brasileira naquele período ${ }^{16}$.

A análise estatística utilizou uma metodologia descritiva. Para amostra paramétrica, utilizou-se média, desvio padrão e moda.

\section{Resultados}

\section{Características da população}

Foram estudadas I 34 crianças atendidas no Serviço de Transplante de Medula Óssea do Hospital de Clínicas da Universidade Federal do Paraná com diagnóstico de anemia aplástica severa desde o ano de 1979 a 1993. Do total de
I34 pacientes, 68 foram submetidos a transplante de medula óssea, 15 realizaram imunossupressão e I 3 faleceram antes de serem submetidosaalgum tratamento(Tabelal).

A população foi dividida em 64 crianças do sexo masculino e 70 crianças do sexo feminino, conforme Figura I. Dos 134 casos documentados, a idade variou de 2 a 18 anos $(X=$ $10,89 \pm 4,48$ anos). A moda foi de 12 anos (Tabela 2, Figuras I e 2).

Dos 134 casos, 76 crianças eram proveniente da região sul, 33 crianças da região sudeste, 13 da região nordeste, nove da região centro-oeste e três da região norte (Tabela3).

Dos 79 familiares que preencheram a ficha, 53 pertenciam a zona urbana $(70,79 \%)$ e 26 pertenciamazonas rurais (29,21\%)-(Figura 3).

Em termos de etiologia, $53 \%$ identificaram um fator causal (Tabela 4). Os pesticidas agrícolas foram relatados por 33 famílias, seguido dos derivados de benzeno que foram relatados em II famílias. Dos 33 familiares que relataram contato com os agrotóxicos, 10 relataram outros fatores causais (três crianças tinham utilizado cloranfenicol, cinco tiveram contato com derivados de benzeno, uma criança com história pregressa de mononucleose infecciosa e uma criança tinha utilizado anticonvulsivante (Tabela5).

\section{Nível socioeconômico}

Das 79 famílias interrogadas, $13,9 \%$ das mães eram analfabetas e $70,9 \%$ não tinham 0 primeiro grau completo -somatório das mães analfabetas edas mães que tinham primeirograu incompleto (Tabela6). A grande maioria das famílias (83,5\%) tinha uma renda familiar "per capita” inferior a um salário mínimo (Tabela 7).

\section{Discussão}

Aimportância de conhecermos determinadas características de pacientes que desenvolveram anemia aplástica severa nos diversos locais está no fato de que elas poderão contribuir na identificação dos mecanismos responsáveis pelo aparecimento da doença, assim como identificar a população mais susceptível em desenvolvê-la.

Neste estudo, conseguimos reavaliar o cadastro de 134 crianças com a doença e adquirir informações complementares através da aplicação de um questionário em 79 famílias contactadas. Este é um número significativo de crianças com esta doença atendida em único serviço. $\mathrm{Na}$ literatura, poucos são os estudos sobreaanemiaaplásticaque focalizemespecificamente a faixa etária pediátrica. Alter etal. estudaram 94 crianças, demonstraram um pico de incidência de 3-5 anos, sendo que a etiologia não foi identificada em $50 \%$ dos pacientes. Não houve diferença em relação ao sexo ${ }^{17}$. Halpérin et al. revisaram 36 crianças comanemia aplásticasevera. A média das idades foi de 7,5 anos, não houve diferença da distribuição da doençaem relação ao sexo, foi identificado o fator causal em dois ca$\operatorname{sos}^{18}$. Clausen realizouumaanálise retrospectiva de todas as crianças com anemia aplástica severa no período de 1967-1982, na Dinamarca. Nesse período, estudou 39 crianças com faixa etária variando 0 - I4 anos. Aincidência da doençafoide $2,2 \operatorname{casos} / 10^{6}$. Não houve predominância de nenhum grupo etário. A relaçãa sexo masculino: feminino foi de 2:I; e 16 pacientes não tinham a forma severa da doença ${ }^{19}$.

Observa-se uma variação na distribuição da doença em relação ao sexo e idade, entre os diversos inquéritos epidemiológicos. Esta variação parece refletir o tipo de população que foi exposta aos agentes mielotóxicos. Na nossa população houve leve predominância do sexo feminino, ea média dasidades foide 10,89 anos.

Houve predomínio também das crianças que vieram da zona urbana da região sul do país, distribuição semelhante aos dados da população geral do Brasil ${ }^{20}$. Questiona-se a probabilidade de que esta também seja a população com maior facilidade de entrar em contato com o serviço de referência.

A relação entre exposição aos fatores etiológicos e o desencadeamento da doença ainda não está muita bem esclarecida. Sabe-se que este processo pode ocorrer por mecanismos diversos (reação de idiossincrasia, mecanismo auto-imune, toxicidade dose-dependente). Tanto na toxicidade dose-dependente como na toxicidade que não foi dose-dependente (reação idiossincrásica), o mecanismo auto-imune poderá estar presente. Após exposição a um fator agressor, na maioria dos casos, inicia-se a liberação por parte de linfócitos ativados, de fatores como gama interferon, que age diretamente inibindo a hematopoiese, assim como outros fatores inibidores como fator de necrose tumoral. Tanto gama interferon como fator de necrose tumoral, inibem a hematopoiese, e iniciam um mecanismo de apoptose das células primordiais hematopoiéticas. Por todos esses fatos, atualmente tem-se uma interpretação que, 
Tabela I - Total dos pacientes do estudo conforme tratamento e resposta a ficha clínica

\begin{tabular}{lcccc}
\hline Tratamento & Responderam a ficha & Não responderam a ficha & Total & $\%$ \\
Transplante & 51 & 17 & 68 & 50,74 \\
Imunossupressão & 15 & 15 & 30 & 22,39 \\
SemTratamento & 13 & 23 & 36 & 26,87 \\
Total & 79 & 55 & 134 & 100,0 \\
\hline
\end{tabular}

Figura I - Distribuição dos casos segundo sexo $(n=\mid 34)$

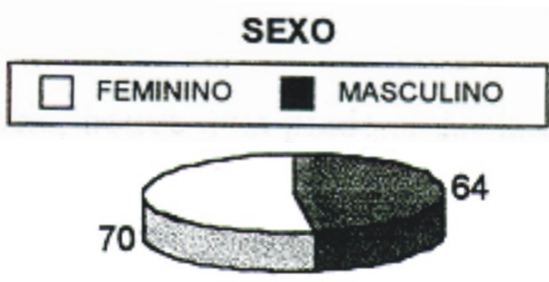

Tabela 2 - Idade dos pacientes com anemia aplástica severa

\begin{tabular}{lcccc}
\hline Classe & $\mathrm{N}$ & $\mathrm{X} \pm \mathrm{DP}$ & Moda & Variância \\
TMO & 68 & $11.31 \pm 4.30$ & 13 & $2-18$ \\
Imunossupressãol & 17 & $10.33 \pm 4.76$ & 6 & $4-18$ \\
Imunossupressão2 & 13 & $9.07 \pm 9.00$ & 6 & $2-17$ \\
Semtratamento & 36 & $10.72 \pm 4.55$ & 12 & $3-18$ \\
Total & 134 & $10.89 \pm 4.48$ & 12 & $2-18$ \\
\hline
\end{tabular}

Imunossupressãol:Ciclosporina+Predinisona - Imunossupressão2:Globulina anti-timocítica

Figura 2 - Poligono de frequiência de idade

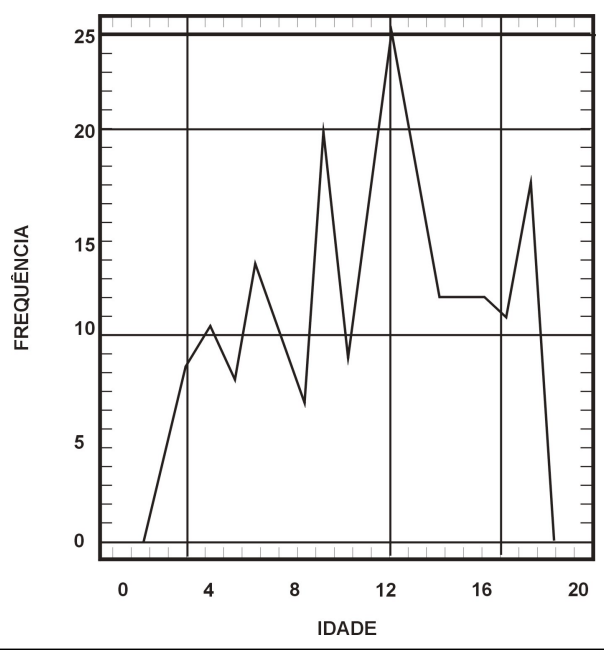

indubitavelmente, a reação imune é um dos fatores mais importantes atuando no quadro de falência medular encontrada na anemia aplástica ${ }^{2 !}$.

O intervalo médio entre a exposição ao agente agressor e o início da doença é pouco conhecido, dificultando os estudos que investigam a etiologia da doença. Os resultados destes estudos são questionados pela possibilidade de viés de memória. Esta possibilidade aumenta quando se trata de um estudo retrospectivo. Além do viés de memória, outra preocupação seria o viés de ruminação - quando o entrevistado lembra com maior propriedade de certas exposições por ficar tentando identificar as causas de sua doença ${ }^{22}$. Essas observações são importantes para termos uma visão crítica do trabalho, porém, mesmo com esta discussão, é interessante comentar os resultados deste estudo.

Chama a atenção a grande quantidade de famílias que relataram a exposição aos pesticidas agrícolas comofator causal.Arelaçãodeexposição aos pesticidas agrícolas eanemia aplástica é muito controversa. Estudoscaso-controlenaFrançaenos EstadosUnidosnão confirmaramestarelação ${ }^{23,24}$. $\mathrm{Na}$ Tailândia, utilizandotambémestudo caso-controle, demonstrou-seumaforteassociação da doença com atividades agrícolas (produtores de grãos), porém não com o uso de pesticidas ${ }^{25}$. No Brasil, umestudo caso-controle, realizado noestado do Paraná, encontrou uma associação com o uso de pesticidas de uso doméstico do grupo do organofosforadocomaanemiaaplásticaadquirida ${ }^{5}$. Outros relatos de caso também no Brasil, relatam uma associação com pesticidas agrícolas com a anemia aplástica ${ }^{26,27}$. Fleming e Timmeny, após uma revisão de relatos de caso encontrados na literatura, colocam a importância de realizar estudos em áreas com alta exposição a pesticidas como nos países não desenvolvidos das áreas tropicais do planeta ${ }^{28}$.

O segundo agente causal relacionado com a anemia aplástica neste estudo foi os produtos derivados de benzeno. $O$ benzeno é um agente queregularmenteproduzfalênciamedular. Dois mecanismos são responsáveis pela sua mielotoxicidade. O primeiro envolve metabólitos hepáticos do benzenofenol, catecolehidroquinona. O segundo envolve um metabólito encontrado após a abertura do anel benzênico. O citocromo P4502EI é responsável em metabolizar o benzenoemfenol, sendo esteummecanismo primário importante de toxicidade. A medula óssea é rica em atividade da enzima peroxidase, enzima responsável por ativar estes metabólitos a reagir com as quinonas, ocasionando uma alteração na diferenciação celular na medula óssea, assim como uma indução do mecanismo de apoptose das células progenitoras ${ }^{29}$. Um componente imunológico do benzeno também tem sido relatado. Existe uma depressão imune por uma linfotoxicidade induzida pelo benzeno, além de uma redução de lgAe lgGemindivíduos cronicamente expostos. Todas essas alterações levama uma depleção da população das células progenitoras da medula óssea ${ }^{30}$. 


\begin{tabular}{ccc}
\hline \multicolumn{3}{c}{ Tabela 3 - Procedência dos pacientes encaminhados com anemia } \\
aplástica severa segundo Estado e Regiäo do Brasil \\
\hline PROCEDÊNCIA & $N^{\circ}$ & $\%$ \\
REGIÄO SUL & 76 & 56,7 \\
Paraná & 39 & 29,1 \\
RioGrande doSul & 20 & 14,9 \\
SantaCatarina & 17 & 12,7 \\
REGIÄO SUDESTE & 33 & 24,6 \\
SäoPaulo & 16 & 11,9 \\
MinasGerais & 16 & 11,9 \\
Riodejaneiro & 01 & 0,8 \\
REGIÃO NORTE & 03 & 2,2 \\
Rondônia & 03 & 2,2 \\
REGIÄO NORDESTE & 13 & 9,7 \\
Piaúl & 01 & 0,8 \\
Pará & 01 & 0,8 \\
Bahia & 05 & 3,7 \\
Pernambuco & 03 & 2,2 \\
Ceará & 02 & 1,4 \\
Maranhão & 01 & 0,8 \\
REGIÃO CENTRO-OESTE & 09 & 6,7 \\
MatoGrosso & 02 & 1,5 \\
MatoGrossodoSul & 03 & 2,2 \\
Goiás & 01 & 0,8 \\
DistritoFederal & 03 & 2,2 \\
TOTAL & 134 & 100 \\
\hline
\end{tabular}

Figura 3 - Distribuição dos pacientes com anemia aplástica severa conforme procedência $(n=79)$

\section{ZONA}

\section{RURAL $\square$ UURBANA}

$29.21 \%$

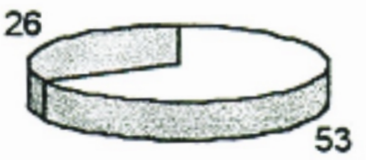

É importante salientar que, por se tratar de um estudo pediátrico, a presença de pesticidas agrícolas e derivados de benzeno como agentes mais citados como prováveis responsáveis pela etiologia da anemia aplástica, demonstra uma exposição indevida dessas crianças a essas substâncias, seja pela exploração da mão de obra infantil, sejapelaimprudêncianoarmazenamento e manipulação desses produtos.

Outros fatores causais como cloranfenicol, anticonvulsivantes, hepatite e mononucleose infecciosa aparecem como uma relação semeIhante ao da literatura ${ }^{31}$.

A associação da anemia aplástica com baixo nível socioeconômico foi descrita pela primeira vez na Tailândia ${ }^{7}$. Esta associação explicaria em aplástica. Os estudos descritivos poderão identificar possíveis fatores de risco para a doença a fim de se testar hipóteses específicas posteriormente. Torna-se urgente realizar um estudo caso-controle, incluindo centros de diferentes regiões do país, para que possamos identificar um perfil da doença no Brasil.

\section{SUMMARY}

SeVERE apLastic anemia in CHILdREN

A Severe Aplastic Anemia is a rare disease that happens in the entire world. Because the rarity, it is difficult to characterize the features of the patients that have this disease, and to find theirpossible etiological factors.

PURPOSE. Toreportall cases ofsevereaplastic anemiainyoung people whowereattended in the hospital and characterize some variables like age, sex, geographicarea of residence, etiologies and socioeconomicstatus.

METHODS. It was donea descriptive analyze of all pediatric patient's features (below 18 years) who came to the hospital in 1979-1993 with severe aplastic anemia based on the classification proposed for Camitta et al. The data were obtained from the hospital records in 134 children, and the investigator in 79 families performedinterviews.

RESULTS. The age ranged from $2-18$ years (mean 10.89 years). The male to female ratio was 64:70. Most of the patients came from urban zone of south Brazil. We did not identify any etiologic agents in $34 \%$ of the cases. Prior exposure to the agricultural pesticides and benzene derivative was the most common etiologic factors that were related. The household income of $83.5 \%$ of the families was $<\$ 65$ U.S./capita, and $70.9 \%$ of the patient's mother did not finish the primaryschool

CONCLUSION. In this group, severe aplastic anemia is more frequent in II years old girls. Most of them are the in urban zone of south Brazil, with low socioeconomic status, and related prior exposure agricultural pesticides and benzene derivative. [Rev Assoc Med Bras 2002; 48(3): 263-7].

KEY WORDS: Severe aplastic anemia. Etiology. Socioeconomic status. Brazil.

\section{RefERÊNCIAS} não confirmou esta relação $0^{5}$

Todos estas observações denotam a dificuldade de avaliar resultados que se correlacionam com uma doença rara e letal como é anemia 
Tabela 4 - Distribuição dos casos que identificaram fatores etiológicos

\begin{tabular}{lcc}
\hline Suspeitas etiologicas & $N^{0}$ de pacientes & $\%$ \\
Comsuspeitas etiológicas & 12 & 53 \\
Semsuspeitas etiológicas & 23 & 29 \\
Informaçãoignorada & 14 & 18 \\
Total & 79 & 100 \\
\hline
\end{tabular}

Tabela 5 - Suspeitas etiológicas dos pacientes com anemia aplástica severa do total de 79 pacientes

\begin{tabular}{lcc}
\hline Suspeitas etiológicas & Número de pacientes & \% do total de pacientes \\
PesticidasAgricolas & 33 & 42 \\
DerivadosdeBenzeno & 11 & 14 \\
Cloranfenicol & 05 & 6 \\
Anticonvulsivante & 01 & 1 \\
Mononucleoseinfecciosa & 01 & 1 \\
Hepatite & 01 & 1
\end{tabular}

* IOpacientes tiveram suspeitas etiológicas combinadas

Tabela 6 - Distribuição da escolaridade materna na população estudada $(n=79)$

\begin{tabular}{llc}
\hline Nível de escolaridade & $N^{\circ}$ & $\%$ \\
Analfabeto & 11 & 13,9 \\
PrimeiroGrauincompleto & 45 & 57,0 \\
PrimeiroGrau & 11 & 13,9 \\
SegundoGrau & 05 & 6,3 \\
TerceiroGrau & 07 & 8,9 \\
TOTAL & 79 & 100 \\
\hline
\end{tabular}

Tabela 7 - Distribuição da renda familiar em salário mínimo "per capita" na população estudada $(n=79)$

\begin{tabular}{llc}
\hline Renda familiar & $N^{\circ}$ & $\%$ \\
$<$ I Salário Mínimo/Capita & 66 & 83,5 \\
$\geq$ Salário Mínimo/Capita & 13 & 16,5 \\
Total & 79 & 100 \\
\hline
\end{tabular}

2. Issaragrisil S, Sriratanasatavorn C, Piankijagum A. Incidence of aplastic anemia in Bangkok. Blood 1991; 77:2166-8

3. Kaufman DW, Kelly JP, Shapiro S. The study experience. In: Kaufman DW, KellyJP, Shapiro S. The drug etiology of agranulocytosis and aplastic anemia. New York: Oxford University Press; 1991. p. 107-13.

4. Young NS, Alter BP. Epidemiology of acquired aplastic anemia. In: Young NS, Alter BP. Aplastic anemia: acquired and inherited. Philadelphia:WB Saunders; 1994. p.24-31.

5. Maluf E. Epidemiologia da anemia plástica severa adquirida-um estudo caso-controle realizado no Brasil. Curitiba; 200 I.

6. Gordon-Smith EC, Issaragrisil S. Epidemiology of aplastic anemia. Baillières Clin Haematol 1992; 5:475-91.

7. Issaragrisil S, Kaufman DW, Anderson TE, Chansung K, Thamprasit T, Sirijirachai J et al. An association of aplastic anemia in Thailand with low socioeconomic status.
Br J Haematol I 995; 91:80-4. Aplastic anaemia study group. Incidence and non-drug aetiologies of aplastic anemia in Thailand. Eur J Haematol 1996; 57:3 I-4.

9. Young NS, Alter BP. Drugs and chemicals. In: Young NS, Alter BP. Aplastic anemia acquired and inherited. Philadelphia: WB Saunders; 1994. p.24-3I.

10. Issaragrisil S, Kaufman D, Thongput A, Chansung K, Thamprasit T, Piankijagum Aet al. Association of seropositivity for hepatitis viruses and aplastic anemia in Thailand. Hepatology 1997; 25: I 255-7.

II. Levy M, Kelly JP, Kaufman DW, Shapiro S. Risk of agranulocytosis and aplastic anemia in relation to history of infectious mononucleosis: a report from the international agranulocytosis and aplastic anemia study. Ann Hematol 1993; 67:187-90

12. Brown KE, Tisdale J, Barret J, Dunbar CE, Young NS. Hepatitis-associated aplastic
8. Issaragrisil S, Kaufaman DW, Anderson TE. anemia. N Engl J Med I 997; 336: 1059-64.

13. Young NS, Mortimer P. Viruses and bone marrow failure. Blood 1 984; 63:729-37.

14. Alter BP, Young NS. The bone marrow failure syndromes. In: Nathan DG, Oski FA. Hematology of infancy and childhood. Philadelphia:WB Saunders; 1994. P I97-216.

15. CamittaB, Thomas ED, Nathan DG. A prospective study of androgens and bone marrow transplantation for treatment of severe aplastic anemia. Blood 1979; 53:504-I 4 .

16. Boletim DIEESE-(Departamento Intersindical de Estatística e Estudos Sócio-Econômicos). São Paulo; 1986.

17. Alter BP, Potter NU, Li FP. Classification and etiology of aplastic anemias. Clin Haematol 1978; 7:431-65.

18. Halpèrin, S, Grisaru D, Freedman MH, Saunders F. Severeacquired aplastic anemia in children: I Iyear experience with bone marrow transplantation and immunosuppressive therapy. Am J Pediatr Hematol Oncol I 989; I I:304-9.

19. Clausen, N. A population study of severe aplastic anemia in children: incidence, etiology and course. Acta Paediatr Scand 1986; 75:58-63.

20. Fundação Instituto Brasileiro de Geografia e Estatística-IBGE. Anuário Estatístico do Brasil; 1992.

21 . Young NS. Immune pathophysiology of acquired aplastic anemia. Eur J Haematol 1996; 57:55-9;

22. Rothman KJ. Modern epidemiology. Boston: Little, Brown and Co; 1986. p.5 I4-7.

23. Mary JY, Baumelou E, Guiguet M, French Cooperative Group for Epidemiological Study of Aplastic Anemia. A prospective multicentric study. Blood 1990; 75: 1646-53.

24 . Wang H, Grufferman S. Aplastic anemia and occupational pesticide exposure: a casecontrol study. J Occup Med I 98I; 23:364-6.

25. Issaragrisil S, Chansung K, Kaufman DW. Aplastic anemia in rural Thailand: its association with grain farming and agricultural pesticide exposure. Am J Public Health 1997; 87: I55 I-5.

26. Lorand I, Souza C, Costa F. Haematological toxicity associated with agricultural chemicals in Brazil. Lancet I 984, I:404.

27. Lorand I, Souza C, Caliani J, Fabron Junior A. Aplasia medular: critérios diagnósticos, evolução clínica e fatores prognósticos. AMB Rev Assoc Méd Bras 1983; 29:215-8.

28. Fleming L, Timmeny W. Aplastic anemia and pesticides. An etiologic association ?J Occup Med 1993; 35: I 106-16.

29. Ross,D. Metabolic basis of benzene toxicity. Eur J Haematol I 996; 57: I I I-8.

30. SmithMT. Overview of benzene-induced aplastic anaemia. Eur J Haematol 1996; 57:107-10.

31. Pasqüini R. Transplante de medula óssea na Anemia Aplástica Severa: estudo de 108 casos, Curitiba, 1991. (tese). Setor de Ciências da Saúde, Universidade Federal do Paraná.

Artigo recebido: |4/ | |/200| Aceito para publicação: 08/04/2002 